\title{
Metabolic Syndrome Is Associated with Aggressive Prostate Cancer Risk Regardless of Race
}

Lourdes Guerrios-Rivera ( $\square$ lourdes.guerrios1@upr.edu )

VA Caribbean Healthcare System https://orcid.org/0000-0003-3846-5105

Lauren Howard

Duke-NUS: Duke-NUS Medical School

\section{Emily Wiggins}

Durham VAMC: Durham VA Medical Center

\section{Cathrine Hoyo}

NC State: North Carolina State University

Delores G. Grant

North Carolina Central University

Jay H. Fowke

University of Tennessee College of Medicine: The University of Tennessee Health Science Center College of Medicine

Tyler R Erickson

Durham VAMC: Durham VA Medical Center

Alexis R. Freedland

UC Irvine: University of California Irvine

Adriana C. Vidal

Cedars-Sinai Medical Center

Stephen J. Freedland

Cedars-Sinai Comprehensive Cancer Center: Cedars-Sinai Medical Center Samuel Oschin Comprehensive Cancer Institute

\section{Research Article}

Keywords: Prostate Carcinoma, Insulin Resistance Syndrome, Outcomes, Black Race, Prevention.

Posted Date: February 17th, 2022

DOI: https://doi.org/10.21203/rs.3.rs-1135975/v1

License: (1) This work is licensed under a Creative Commons Attribution 4.0 International License. Read Full License 


\section{Abstract \\ Purpose}

A recent meta-analysis suggested a link between Metabolic Syndrome (MS) and high-grade prostate cancer (PC), though few black men were included. We tested the link between MS and PC risk in a population of black and white men undergoing prostate biopsy. We hypothesized MS would be linked with aggressive PC, regardless of race.

\section{Methods}

Among men undergoing prostate biopsy at the Durham Veterans Affairs Hospital, we abstracted history of or treatment for hypertension ( $\geq 130 / 85 \mathrm{mmHg}$ ), dyslipidemia ( $\mathrm{HDL}<40 \mathrm{mg} / \mathrm{dL}$ ), hypertriglyceridemia ( $\geq 150 \mathrm{mg} / \mathrm{dL}$ ), diabetes/impaired fasting glucose (fasting glucose $\geq 100 \mathrm{ml} / \mathrm{dL}$ ), and obesity (waist circumference $\geq 40$ inches) in the year prior to biopsy. Biopsy grade group (GG) was categorized as low (GG1) or high-grade (GG2-5). Multinomial logistic regression was used to examine MS (3-5 components) vs. no MS (0-2 components) and risk of high-grade and low-grade vs. no PC adjusting for key confounders. Interactions between race and MS were tested.

\section{Results}

Of 1,051 men (57\% black), 532 (51\%) had MS. Men with MS were older, more likely to be non-black, and had larger prostate volumes (all $\mathrm{p} \leq 0.011)$. On multivariable analysis, $\mathrm{MS}$ was associated with high-grade $\mathrm{PC}(\mathrm{OR}=1.73,95 \% \mathrm{Cl} 1.21-2.48, \mathrm{p}=0.003)$, but not overall $P C$ $(\mathrm{OR}=1.17,95 \% \mathrm{Cl} 0.88-1.57, \mathrm{p}=0.29)$ or low-grade $(\mathrm{OR}=0.87,95 \% \mathrm{Cl} 0.62-1.21, \mathrm{p}=0.39)$. Results were similar in black and non-black men (all $\mathrm{p}$ interactions $>0.25$ ).

\section{Conclusion}

Regardless of race, MS was associated with aggressive PC, but not overall PC risk. If confirmed in other studies, our data suggest that prevention of MS may reduce the risk of developing aggressive PC in both black and non-black men.

\section{Introduction}

Metabolic Syndrome (MS) prevalence is increasing and is a serious public health problem worldwide.[1, 2] In the US, MS affects more than one-third of adults.[1] MS consists of a diagnosis of at least three of the following five risk factors: increased abdominal obesity, dyslipidemia (high triglycerides and high HDL cholesterol), hypertension, and abnormal glycemic status.[3] Given obesity, a MS component, is linked with multiple cancers,[4] it is plausible MS may also be associated with cancer development and progression. Indeed, MS has been linked with numerous cancers, including increased PC risk.[5] A recent meta-analysis of 130,000 men found that MS is associated with increased PC incidence,[6] though the overall increased risk was modest. However, not all individual studies were consistent with some finding MS was associated with lower PC risk[7, 8] Nonetheless, the meta-analysis found MS was strongly linked with high-grade $P C(O R=1.89, p<0.0001)$. [6]

Importantly, nearly all studies evaluating MS and PC risk focused on White, European, or Asian men.[9-11] Whether results differ by race and specifically whether MS is linked with PC among black men, who have one of the highest PC incidence and mortality rates in the world, is limited.[12] To date, few studies examined the link between MS and PC in Black men.[12, 13]

To evaluate the link between MS and PC risk across different races, we analyzed a case-control study of black and non-black (predominantly white) men undergoing prostate biopsies at the Durham Veterans Affairs (VA) Medical Center (DVAMC) in Durham, North Carolina. We choose an equal access healthcare setting to minimize potential effects that access to care may have on our results. Given obesity, which is one of the components of MS, is linked with lower PSA,[14] but larger prostate sizes,[15] it is noteworthy that all men in this biopsy cohort had data available on PSA and prostate volume to adjust for in the analyses to account for potential differences between men with and without MS. Furthermore, obesity and insulin resistance have been recognized as the leading contributors in MS, which in turn is associated with the production of various pro-inflammatory cytokines which may promote genomic instability and a greater risk of cancer development.[16] Likewise, in the Reduction by Dutasteride of Prostate Cancer Events (REDUCE) clinical trial, high cholesterol levels, another MS component, were associated with high-grade PC but not overall nor low-risk PC.[17] Based upon the recent meta-analysis[6] and our understanding we were unlikely to be powered to detect a very small increase in total PC incidence, we a priori hypothesized that MS would be linked with aggressive $P C$ regardless of race, but not overall PC incidence. 


\section{Methods \\ Study Design}

Data were obtained from an ongoing case-control study of veterans undergoing prostate biopsy for concerns about PC at the DVAMC. The study was approved by the institutional review board and written informed consent was obtained from all subjects. Subjects were recruited between January 2007 and July 2018 from the urology clinic. Eligible subjects were men with no prior PC history undergoing a prostate biopsy because of abnormal PSA and/or suspicious digital rectal exam (DRE) as clinically indicated. For men with multiple biopsies performed at the DVAMC, we only used data from their initial biopsy at the DVAMC. Of 2279 eligible subjects, 1322 consented to participate (58\% response rate). We excluded 249 subjects due to missing PSA, DRE, prostate volume, MS data, or grade group, and 22 active surveillance biopsies as these subjects would have been diagnosed with PC, resulting in 1051 men.

MS was defined as three or more of the following: dyslipidemia, hypertriglyceridemia, diabetes/impaired fasting glucose, hypertension, and abdominal obesity. Dyslipidemia was defined as $\mathrm{HDL}<40 \mathrm{mg} / \mathrm{dL}$ in the year prior to biopsy. Hypertriglyceridemia was defined as triglycerides $\geq 150 \mathrm{mg} / \mathrm{dL}$ or prescription drugs to treat high triglycerides in the year prior to biopsy. Diabetes was defined as fasting glucose $>100 \mathrm{ml} / \mathrm{dL}$, prescription of an anti-diabetic agent, or diagnosis of diabetes in the medical record in the year prior to biopsy. Hypertension was defined as two blood pressure measurements where systolic blood pressure $>130 \mathrm{mmHg}$ or diastolic blood pressure $>85 \mathrm{mmHg}$ within the year prior to biopsy. Waist circumference was measured by trained personnel and obesity was defined as waist circumference $\geq 40$ inches. Race was selfreported as black, white, Asian/Pacific Islander, or American Indian/Alaska Native. As $<1 \%$ of patients were races other than black or white, race was grouped as black or non-black with $99 \%$ of the non-black men being white. Prostate volume was measured at biopsy via transrectal ultrasound. Biopsy grade was categorized as low-grade (grade group 1) or high-grade (grade group 2-5).

We tested the association between MS (categorical, no: 0-2 vs. yes: 3-5 components), demographic and clinical variables using chi-squared for categorical variables and Wilcoxon rank sum for continuous variables. Variables included age (continuous), year (continuous), race (black vs. non-black), baseline PSA (continuous), DRE (normal vs. abnormal) and pre-study prostate volume (continuous). In an exploratory analysis, we examined variables by both MS and race using chi-squared or Kruskal-Wallis tests.

Logistic regression was used to examine the association between MS and PC risk versus no PC. Multinomial logistic regression was used to examine the association between MS and risk of low-grade PC (GG 1) versus no PC and high-grade PC (GG 2-5) versus no PC. The number of MS components was also tested in the above models as a continuous variable. Multivariable models were adjusted for age, year of consent, race, log-transformed baseline PSA, DRE, and log-transformed prostate volume. The interaction between race and MS in adjusted analyses was also tested and models were stratified by race. In secondary analyses, the association between each individual MS component and PC risk and grade was examined in separate univariable and multivariable models, and interactions were tested between race and each component. As a sensitivity analysis, we then repeated all analyses defining low-grade PC as GG 1-2 and high-grade PC as GG 2-5.

Significance was defined as $\mathrm{P}<0.05$. All analyses were performed using SAS 9.4 (SAS Institute; Cary, NC).

\section{Results}

\section{Baseline characteristics}

Among 1051 men, 532 (51\%) had MS and 519 (49\%) did not. Having MS was associated with older age, non-black race, and larger prostate volume (Table 1, all $\mathrm{p} \leq 0.011$ ). There was no association between MS with PSA or DRE.

Black men were younger at biopsy, had higher PSA, fewer suspicious DREs, and higher rates of overall cancer (all $p \leq 0.015$; Supplementary Table 1). Non-black men with MS had the largest prostate volumes, while black men without MS had the lowest prostate volumes ( $\mathrm{p}<0.001)$.

\section{MS and PC outcomes}

While MS was not associated with overall PC (52\% vs. 55\%, p=0.27), MS was associated with PC grade $(p=0.005$, Table 1$)$. Specifically, men with MS were less likely to have low-grade ( $20 \%$ vs. $28 \%$ ) and more likely to have high-grade PC (32\% vs. $27 \%)$ or no PC (48\% vs. $45 \%)$.

On unadjusted analyses, MS was not associated with overall PC (OR=0.87, 95\% Cl 0.68-1.11, p=0.26) or high-grade $\mathrm{PC}(\mathrm{OR}=1.10,95 \% \mathrm{Cl} 0.83-$ $1.46, \mathrm{p}=0.52$, Table 2). However, MS was associated with lower odds of low-grade PC $(\mathrm{OR}=0.65,95 \% \mathrm{Cl} 0.48-0.89, \mathrm{p}=0.003)$. On multivariable analysis, MS was associated with increased odds of high-grade PC (OR=1.73, 95\% Cl 1.21-2.48, $\mathrm{p}=0.003$; Table 2), but not overall (OR=1.17, $95 \% \mathrm{Cl} 0.88-1.57, \mathrm{p}=0.29)$ or low-grade PC $(\mathrm{OR}=0.87,95 \% \mathrm{Cl} 0.62-1.21, \mathrm{p}=0.39)$. When low-grade was treated as the reference, $\mathrm{MS}$ was associated with increased odds of high-grade PC vs. low-grade PC $(O R=2.00,95 \% \mathrm{Cl} 1.38-2.89, p<0.001)$. 


\begin{tabular}{|c|c|c|c|}
\hline & No MS $(N=519)$ & MS (N=532) & $\mathrm{p}$ value \\
\hline Age at biopsy & & & $0.011^{1}$ \\
\hline Median & 63 & 64 & \\
\hline Q1, Q3 & 59,67 & 60,68 & \\
\hline Year & & & $0.057^{1}$ \\
\hline Median & 2011 & 2011 & \\
\hline Q1, Q3 & 2009, 2016 & 2009, 2016 & \\
\hline Race & & & $<0.001^{2}$ \\
\hline Non-black & $184(35 \%)$ & $265(50 \%)$ & \\
\hline Black & $335(65 \%)$ & 267 (50\%) & \\
\hline PSA level, ng/ml & & & $0.64^{1}$ \\
\hline Median & 5.9 & 5.8 & \\
\hline Q1, Q3 & $4.5,8.6$ & $4.6,8.2$ & \\
\hline Prostate volume, ml & & & $<0.001^{1}$ \\
\hline Median & 39.0 & 43.0 & \\
\hline Q1, Q3 & $27.3,54.5$ & $31.3,62.1$ & \\
\hline DRE & & & $0.65^{2}$ \\
\hline Not suspicious for cancer & $384(74 \%)$ & $387(73 \%)$ & \\
\hline Suspicious for cancer & $135(26 \%)$ & $145(27 \%)$ & \\
\hline Hypertension & $399(77 \%)$ & $496(93 \%)$ & \\
\hline Hypercholesterolaemia & $70(13 \%)$ & $355(67 \%)$ & \\
\hline Hypertriglyceridemia & $55(11 \%)$ & $338(64 \%)$ & \\
\hline Diabetes & $60(12 \%)$ & $274(52 \%)$ & \\
\hline Obesity (waist $\geq 40$ inches) & $186(36 \%)$ & $467(88 \%)$ & \\
\hline Biopsy had cancer (yes/no) & & & $0.27^{2}$ \\
\hline No Cancer & $232(45 \%)$ & $256(48 \%)$ & \\
\hline Cancer & $287(55 \%)$ & $276(52 \%)$ & \\
\hline PC Grade & & & $0.005^{2}$ \\
\hline No cancer & $232(45 \%)$ & $256(48 \%)$ & \\
\hline Low-grade (grade group 1) & $146(28 \%)$ & $105(20 \%)$ & \\
\hline High-grade (grade group 2-5) & $141(27 \%)$ & $171(32 \%)$ & \\
\hline${ }^{1}$ Wilcoxon ${ }^{2}$ Chi-Square & & & \\
\hline
\end{tabular}

Table 2. Univariable and multivariable OR and $95 \%$ Cls for the association between metabolic syndrome and risk of prostate cancer 


\begin{tabular}{|c|c|c|c|c|c|c|c|c|c|}
\hline & \multicolumn{3}{|c|}{ All prostate cancers } & \multicolumn{3}{|c|}{ Low-grade PC } & \multicolumn{3}{|c|}{ High-grade PC } \\
\hline & OR & $95 \% \mathrm{Cl}$ & $P$ & OR & $95 \% \mathrm{Cl}$ & $\mathbf{P}$ & OR & $95 \% \mathrm{Cl}$ & $\mathbf{P}$ \\
\hline \multicolumn{10}{|l|}{ Univariable } \\
\hline No metabolic syndrome & Ref. & - & - & Ref & - & - & Ref. & - & - \\
\hline Metabolic syndrome & 0.87 & $0.68-1.11$ & 0.26 & 0.65 & $0.48-0.89$ & 0.003 & 1.10 & $0.83-1.46$ & 0.52 \\
\hline Continuous & 0.90 & 0.83-0.99 & 0.039 & 0.80 & $0.71-0.90$ & $<0.001$ & 1.00 & $0.89-1.12$ & 0.96 \\
\hline \multicolumn{10}{|l|}{ Multivariable* } \\
\hline No metabolic syndrome & Ref. & - & & Ref. & - & & Ref. & - & \\
\hline Metabolic syndrome & 1.17 & $0.88-1.57$ & 0.29 & 0.87 & $0.62-1.21$ & 0.39 & 1.73 & $1.21-2.48$ & 0.003 \\
\hline Continuous & 1.03 & $0.92-1.16$ & 0.63 & 0.91 & $0.79-1.04$ & 0.15 & 1.20 & $1.04-1.39$ & 0.011 \\
\hline
\end{tabular}

On univariable analysis, greater number of MS components (as a continuous variable) was associated with lower odds of overall PC (OR=0.90 per MS component, 95\% Cl 0.83-0.99, p=0.039; Table 2) and lower odds of low-grade PC (OR=0.80 per MS component, 95\%Cl 0.71-0.90, $\mathrm{p}$ <0.001). Number of MS components was not associated with high-grade PC versus no PC. On multivariable analysis, greater number of MS components was associated with increased odds of high-grade $\mathrm{PC}(\mathrm{OR}=1.20$ per MS component, 95\% $\mathrm{Cl} 1.04-1.39, \mathrm{p}=0.011)$. Associations with overall and low-grade PC in crude analyses were attenuated and no longer significant after multivariable adjustment.

\section{Interaction testing}

We tested the interaction between race and number of MS components in adjusted analyses and found no significant interactions for estimating PC risk ( $p=0.37$ for all cancer, $p=0.91$ for low-grade PC, $p=0.25$ for high-grade PC). When stratified by race (Supplementary Table 2 ), results were generally similar in non-black and black men, although slightly stronger in non-black men, though as noted there were no significant interactions. The association between MS and high-grade PC was stronger in non-black men $(\mathrm{OR}=2.41,95 \% \mathrm{Cl} 1.33-4.36, \mathrm{p}=0.003)$ and did not reach statistical significance in black men $(\mathrm{OR}=1.44,95 \% \mathrm{Cl} 0.91-2.29, \mathrm{p}=0.12)$, though again the interaction test was null ( $\mathrm{p}=0.25)$.

\section{Individual MS components}

On univariable analysis, there was a trend for obesity, hypertriglyceridemia, dyslipidemia, hypertension, and diabetes (in separate models) to be associated with lower odds of low-grade PC (OR range 0.62-0.86), but only obesity, hypertriglyceridemia, and dyslipidemia reached significance (Table 3). The ORs for the individual MS components and risk of high-grade PC were not consistent and ranged from 0.84 to 1.33. On multivariable analysis, ORs for all MS components were all < 1 for low-grade PC (range 0.80 to 0.91 ) but none were significant. In contrast, ORs for each MS component were all >1 for high-grade PC (range 1.17 to 1.55), but only obesity reached significance. All interactions between individual components and race for predicting overall or low/high-grade PC were not statistically significant ( $p \geq 0.18$, Table 3$)$. 
Table 3

Univariable and multivariable OR and $95 \%$ Cls for the association between metabolic syndrome components and risk of prostate cancer

\begin{tabular}{|c|c|c|c|c|c|c|c|c|c|c|c|c|}
\hline \multirow{2}{*}{$\begin{array}{l}\text { Metabolic syndrome } \\
\text { component }\end{array}$} & \multicolumn{3}{|c|}{ All prostate cancers } & \multirow{2}{*}{$\begin{array}{l}\text { Race } \\
\text { interaction }\end{array}$} & \multicolumn{3}{|c|}{ Low-grade PC } & \multirow{2}{*}{$\begin{array}{l}\text { Race } \\
\text { interaction }\end{array}$} & \multicolumn{3}{|c|}{ High-grade PC } & \multirow{2}{*}{$\begin{array}{l}\text { Race } \\
\text { interactior }\end{array}$} \\
\hline & OR & $\begin{array}{l}95 \% \\
\mathrm{Cl}\end{array}$ & $\mathbf{P}$ & & OR & $\begin{array}{l}95 \% \\
\mathrm{Cl}\end{array}$ & $\mathbf{P}$ & & OR & $\begin{array}{l}95 \% \\
\mathrm{Cl}\end{array}$ & $\mathbf{P}$ & \\
\hline \multicolumn{13}{|l|}{ Univariable } \\
\hline Obesity & 0.76 & $\begin{array}{l}0.59- \\
0.98\end{array}$ & 0.032 & & 0.62 & $\begin{array}{l}0.46- \\
0.85\end{array}$ & 0.003 & & 0.90 & $\begin{array}{l}0.67- \\
1.21\end{array}$ & 0.48 & \\
\hline Hypertriglyceridemia & 0.88 & $\begin{array}{l}0.69- \\
1.14\end{array}$ & 0.34 & & 0.72 & $\begin{array}{l}0.52- \\
0.99\end{array}$ & 0.046 & & 1.03 & $\begin{array}{l}0.77- \\
1.38\end{array}$ & 0.82 & \\
\hline Dyslipidemia & 0.74 & $\begin{array}{l}0.58- \\
0.95\end{array}$ & 0.019 & & 0.63 & $\begin{array}{l}0.46- \\
0.87\end{array}$ & 0.005 & & 0.84 & $\begin{array}{l}0.63- \\
1.12\end{array}$ & 0.24 & \\
\hline Hypertension & 1.05 & $\begin{array}{l}0.75- \\
1.47\end{array}$ & 0.79 & & 0.82 & $\begin{array}{l}0.55- \\
1.23\end{array}$ & 0.33 & & 1.33 & $\begin{array}{l}0.87- \\
2.03\end{array}$ & 0.19 & \\
\hline Diabetes & 0.98 & $\begin{array}{l}0.76- \\
1.28\end{array}$ & 0.90 & & 0.86 & $\begin{array}{l}0.61- \\
1.20\end{array}$ & 0.36 & & 1.10 & $\begin{array}{l}0.81- \\
1.48\end{array}$ & 0.56 & \\
\hline \multicolumn{13}{|l|}{ Multivariable* } \\
\hline Obesity & 1.14 & $\begin{array}{l}0.84- \\
1.54\end{array}$ & 0.40 & 0.87 & 0.85 & $\begin{array}{l}0.60- \\
1.21\end{array}$ & 0.54 & 0.80 & 1.55 & $\begin{array}{l}1.06- \\
2.25\end{array}$ & 0.023 & 0.82 \\
\hline Hypertriglyceridemia & 1.02 & $\begin{array}{l}0.76- \\
1.39\end{array}$ & 0.88 & 0.26 & 0.90 & $\begin{array}{l}0.64- \\
1.28\end{array}$ & 0.36 & 0.53 & 1.27 & $\begin{array}{l}0.88- \\
1.83\end{array}$ & 0.20 & 0.28 \\
\hline Dyslipidemia & 0.99 & $\begin{array}{l}0.73- \\
1.33\end{array}$ & 0.94 & 0.38 & 0.84 & $\begin{array}{l}0.59- \\
1.18\end{array}$ & 0.31 & 0.72 & 1.21 & $\begin{array}{l}0.84- \\
1.74\end{array}$ & 0.31 & 0.29 \\
\hline Hypertension & 0.94 & $\begin{array}{l}0.63- \\
1.40\end{array}$ & 0.74 & 0.18 & 0.80 & $\begin{array}{l}0.52- \\
1.25\end{array}$ & 0.33 & 0.31 & 1.17 & $\begin{array}{l}0.71- \\
1.95\end{array}$ & 0.54 & 0.30 \\
\hline Diabetes & 1.10 & $\begin{array}{l}0.81- \\
1.51\end{array}$ & 0.54 & 0.91 & 0.91 & $\begin{array}{l}0.63- \\
1.30\end{array}$ & 0.59 & 0.87 & 1.39 & $\begin{array}{l}0.95- \\
2.03\end{array}$ & 0.089 & 0.93 \\
\hline
\end{tabular}

\section{Sensitivity Analysis}

Upon reclassification of low-grade (GG 1-2) and high-grade (GG 3-5) PC, there were fewer high-grade PC cases and MS was not statistically associated with PC grade ( $p=0.07$, Supplementary Table 3). We observed no other appreciable differences in overall trends with MS. MS remained associated with increased odds of high-grade PC, unrelated to low-grade PC and had no interactions with race (Supplementary Table 4 \& 5).

\section{Discussion}

MS and PC are both common problems.[2] Despite a recent meta-analysis finding MS was associated with increased PC risk,[6] individual studies are mixed.[7-11] Importantly, the association between MS and PC risk in black men has not been well studied. Given black men are at increased risk of some MS components[18, 19] such as hypertension, obesity, diabetes, and aggressive PC,[20] understanding whether race modifies this association is of utmost importance. We tested the link between MS and PC risk in a black and non-black case-control study of men undergoing prostate biopsy. In the REDUCE study, where most of the participants were Caucasians, all men underwent mandated biopsies regardless of PSA levels, MS was associated with increased risk of high-grade PC but not with overall or low-grade PC,[21] therefore, we hypothesized that MS would not be linked with overall PC risk but would be linked with aggressive PC and results would be similar in both black and non-black men.

Consistent with our hypothesis, MS and individual components were not associated with overall PC risk regardless of race. While on the surface these results differ from the meta-analysis and several papers that found a positive association between MS and total PC risk,[6, 9, 11] it is noteworthy that in the present study the $\mathrm{OR}=1.17$, identical to the $\mathrm{OR}=1.17(95 \% \mathrm{Cl} 1.00-1.36)$ from the meta-analysis.[6] Thus, while our findings for PC risk were not significant, they are consistent with modest increased risk and in-line with prior studies. Given the modest link between MS and overall PC risk, this association is of unclear clinical significance. 
Given practice trends away from trying to diagnose low-grade PC,[22] it is important to also focus on high-grade PC. On unadjusted analyses, there was no association between MS and high-grade PC. However, after adjusting for clinical characteristics, MS was significantly linked with nearly twofold increased odds of high-grade PC. Our results were nearly identical to the recent meta-analysis,[6] that found an $\mathrm{OR}=1.89(95 \% \mathrm{Cl}$ 1.50-2.38, p<0.0001) for high-grade PC. Likewise, other large studies have also found a link between MS and high-grade PC.[9, 21] When we examined individual MS components separately, each component was modestly linked with increased PC risk, but none reached significance. Thus, there was no single MS component that drove the association with high-grade PC, but rather the greater number of components, the greater the risk. Collectively, our results and those of prior studies suggest that MS is a significant risk factor for high-grade PC.[9, 21]

A major gap in the literature, however, is that nearly all prior studies evaluating MS and PC risk focused on either white or Asian men.[10, 11] The association between MS and PC in black men is under-studied.[12] This is a major unmet need as black men are at increased risk for some MS components,[18, 19] and for aggressive PC.[20, 23] We found no evidence for an interaction between MS and race, suggesting results apply equally to black and non-black men, regardless of how we defined high-grade disease. One prior study examined interactions between race and MS for PC risk and found that MS was suggestively predictive of increased PC risk in black men, but not in white men.[12] However, this study only included 378 black men (vs. 613 in the current) and included controls that did not undergo biopsy. Moreover, no formal interaction testing was done in that study, and thus, whether the results truly differed by race is unknown. Clinically, our results are important as the known risk factors for PC are family history, age, and race. Our data support the hypothesis that regardless of race, MS should be considered a risk factor for high-grade PC. Ultimately, whether managing MS via drugs (statins, metformin, etc.) or lifestyle interventions can reduce the risk of high-grade PC warrants further study. [24, 25]

While the biological mechanisms why MS is differentially associated with high-grade PC are not clear, one possible mechanism is the MSassociated pro-inflammatory state.[26] Obesity-linked inflammation leads to altered metabolic signaling with activation of cytokines, imbalance between adipokines along with insulin growth factor-1 (IGF-1) axis expression.[27] This environment induces the production of reactive oxygen and free radicals with subsequent genomic alterations as DNA breaks promoting the transmembrane protease, serine 2 , erythroblast transformation-specific-related gene (TMPRSS2: ERG) gene fusion[28] present in 50\% of PCs.[29] Intriguingly, in tumors with TMPRSS2: ERG gene fusion, obesity is a particularly strong PC risk factor.[30] Unfortunately, as TMPRSS2: ERG gene fusion status is not known in this study, we are unable to verify this. While the TMPRSS2: ERG gene fusion is less common in black men[31] and we found no interaction by race, the strength of the association between MS and high-grade PC was slightly weaker in black men. Whether this association is indeed weaker in black men certainly requires further study, but if true, it is intriguing to speculate that this may, in part, relate to less TMPRSS2: ERG gene fusion positive tumors in black men.

Another MS component, dyslipidemia, appears to be related to PC,[32] with several mechanisms proposed, including accumulation of cholesterol in PC cells membranes allowing pro-carcinogenic cell signaling.[33] Consistent with MS being selectively linked with high-grade PC, we showed in the REDUCE study that high cholesterol was unrelated to overall PC risk, but was associated with high-grade PC.[32] Our study builds on prior studies supporting the need for PC prevention clinical trials to modify MS factors to reduce high-grade PC including both white and black men.

Our study has some limitations. First, our MS definition may differ from the rigorous NCP ATP III definition[3] but is consistent with classic MS definitions in prior studies.[6] Second, our observed null associations for overall PC risk may due to modest sample size and we cannot exclude a modest association between MS and overall PC risk. Third, our findings were based on Veterans in the VA system; whether results apply to men outside the VA system requires further study, though our results were consistent with the meta-analysis[6] which included nearly only men outside the VA system, suggesting that our results do apply outside the VA. Fourth, we lacked data on other key factors associated with MS such as glycemic control, distribution of diabetes type 1 vs type 2, diabetes duration, testosterone, inflammatory markers, physical activity, and diet, known confounders of MS[2] possibly attenuating the ORs in our results. In addition, the definition of PC aggressiveness was grade on biopsy. Though this correlates with long-term PC progression risk,[34] future studies with alternatives definitions (i.e., metastases, stage, and PC death) are recommended. Lastly, the study subjects were men referred for a biopsy due to elevated PSA, abnormal digital exam or both. Therefore, the prevalence of the exposure of MS in the "healthy" control may be different and this impact remains to be determined. Notwithstanding these limitations, our key strength was the use of a contemporary prospective collection of data from men all undergoing biopsy.

\section{Conclusion}

In our study of black and white men undergoing prostate biopsy, we found MS was not associated with overall PC risk but was linked to highgrade PC. Importantly, results were similar in black and non-black men. Given the strength of the data linking MS and high-grade PC, future studies should test whether interventions including lifestyle modifications can reduce the impact of MS on high-grade PC, and merits further investigation in large diverse multiethnic populations. 


\section{Declarations}

\section{Acknowledgments}

LEADS Support; National Institute of General Medical Sciences, R25 GM116740 (LGR)

\section{Funding}

Research Scholar Grant, RSG-18-018-01 - CPHPS, from the American Cancer Society. (AV)

\section{Author contributions}

Included all the authors associated to this manuscript: Lourdes Guerrios-Rivera (LGR) MD, MSc Lauren E. Howard (LEH) MS, Emily K. Wiggins (EKW) MPH, Cathrine Hoyo (CH) PhD, Delores G. Grant (DGG) PhD Jay H. Fowke (JHF), PhD, MPH, Tyler R Erickson (TRE) MS, Alexis R. Freedland (ARF), MS, PhD (c), Adriana C. Vidal (ACV), PhD and Stephen J. Freedland (SJF) MD

Conceptualization; Lourdes Guerrios-Rivera, Lauren E Howard, Stephen JFreedland

Data curation; Lauren E Howard, Tyler RErickson, Emily K Wiggins, Cathrine Hoyo, Delores GGrant, Stephen JFreedland

Formal analysis, Lauren E Howard, Tyler RErickson, Stephen JFreedland

Investigation; Lourdes Guerrios-Rivera, Lauren E. Howard, Emily K. Wiggins, CathrineHoyo, Delores G. Grant, Jay H. Fowke, Alexis R. Freedland, Adriana C. Vidal, and Stephen J. Freedland

Methodology,Lourdes Guerrios-Rivera, Lauren E. Howard, Emily K. Wiggins, CathrineHoyo, Delores G. Grant, Jay H. Fowke, Tyler R Erickson, Alexis R. Freedland, Adriana C. Vidal, and Stephen J. Freedland

Project administration; Lourdes Guerrios-Rivera, Lauren E. Howard, Emily K. Wiggins,Stephen J. Freedland

Writing original draft. Lourdes Guerrios, Lauren Howard, Stephen Freedland.

Writing-Review \& editing: Lourdes Guerrios-Rivera, Lauren E. Howard,Emily K. Wiggins, Cathrine Hoyo, Delores G. Grant,Jay H. Fowke,Tyler R Erickson, Alexis R. Freedland, Adriana C. Vidal'and Stephen J. Freedland

All authors read, critically revised and approved the final manuscript

\section{Ethics Approval}

The study was approved by Durham VA Medical CenterInstitutional Review Board, reference number \#1141.

\section{Consent to participate}

Informed consent was obtained from all individual participants included in the study.

\section{Disclosure of potential conflicts of interest}

The authors have no financial or proprietary interests in any material discussed in this article.

There are no direct or indirect commercial financial incentive associated with publishing the article.

No funding agreement limits my ability to complete and publish this research/study.

\section{Competing Interests}

Dr Stephen J Freedland is Editor-in-Chief, Prostate Cancer and Prostatic Diseases

\section{Data availability}

Authors can confirm that all relevant data are included in the article and/or its supplementary information files.

\section{References}


[1] J. X. Moore, N. Chaudhary, and T. Akinyemiju, "Metabolic Syndrome Prevalence by Race/Ethnicity and Sex in the United States, National Health and Nutrition Examination Survey, 1988-2012," (in eng), Prev Chronic Dis, vol. 14, p. E24, Mar 16 2017, doi: 10.5888/pcd14.160287.

[2] M. G. Saklayen, "The Global Epidemic of the Metabolic Syndrome," (in eng), Curr Hypertens Rep, vol. 20, no. 2, 2018, doi: 10.1007/s11906018-0812-z.

[3] "Executive Summary of The Third Report of The National Cholesterol Education Program (NCEP) Expert Panel on Detection, Evaluation, And Treatment of High Blood Cholesterol In Adults (Adult Treatment Panel III)," (in eng), Jama, vol. 285, no. 19, pp. 2486-97, May 16 2001, doi: 10.1001/jama.285.19.2486.

[4] G. De Pergola and F. Silvestris, "Obesity as a major risk factor for cancer," (in eng), J Obes, vol. 2013, p. 291546, 2013, doi: $10.1155 / 2013 / 291546$.

[5] P. Pothiwala, S. K. Jain, and S. Yaturu, "Metabolic syndrome and cancer," (in eng), Metab Syndr Relat Disord, vol. 7, no. 4, pp. 279-88, Aug 2009, doi: 10.1089/met.2008.0065.

[6] M. Gacci et al., "Meta-analysis of metabolic syndrome and prostate cancer," (in eng), Prostate Cancer Prostatic Dis, vol. 20, no. 2, pp. 146155, Jun 2017, doi: 10.1038/pcan.2017.1.

[7] A. J. Tande, E. A. Platz, and A. R. Folsom, "The metabolic syndrome is associated with reduced risk of prostate cancer," American journal of epidemiology, vol. 164, no. 11, pp. 1094-1102, 2006.

[8] 0. Telli et al., "Does metabolic syndrome or its components associate with prostate cancer when diagnosed on biopsy?," (in eng), Ther Adv Med Oncol, vol. 7, no. 2, pp. 63-7, Mar 2015, doi: 10.1177/1758834014560158.

[9] B. Bhindi et al., "Dissecting the association between metabolic syndrome and prostate cancer risk: analysis of a large clinical cohort," (in eng), Eur Urol, vol. 67, no. 1, pp. 64-70, Jan 2015, doi: 10.1016/j.eururo.2014.01.040.

[10] B. A. Dickerman et al., "Midlife metabolic factors and prostate cancer risk in later life," (in eng), Int J Cancer, vol. 142, no. 6, pp. 1166-73, Mar 15 2018, doi: 10.1002/ijc.31142.

[11] J. Q. Zhang, H. Geng, M. Ma, X. Y. Nan, and B. W. Sheng, "Metabolic Syndrome Components are Associated with Increased Prostate Cancer Risk," (in eng), Med Sci Monit, vol. 21, pp. 2387-96, 2015, doi: 10.12659/msm.893442.

[12] J. L. Beebe-Dimmer et al., "Racial differences in risk of prostate cancer associated with metabolic syndrome," (in eng), Urology, vol. 74, no. 1, pp. 185-90, Jul 2009, doi: 10.1016/j.urology.2009.03.013.

[13] J. L. Beebe-Dimmer, R. L. Dunn, A. V. Sarma, J. E. Montie, and K. A. Cooney, "Features of the metabolic syndrome and prostate cancer in African-American men," (in eng), Cancer, vol. 109, no. 5, pp. 875-81, Mar 1 2007, doi: 10.1002/cncr.22461.

[14] L. L. Banez et al., "Obesity-related plasma hemodilution and PSA concentration among men with prostate cancer," (in eng), JAMA : the journal of the American Medical Association, vol. 298, no. 19, pp. 2275-80, Nov 212007.

[15] S. J. Freedland et al., "Obesity, serum prostate specific antigen and prostate size: implications for prostate cancer detection," The Journal of urology, vol. 175, no. 2, pp. 500-4, Feb 2006.

[16] A. M. De Marzo et al., "Inflammation in prostate carcinogenesis," (in eng), Nat Rev Cancer, vol. 7, no. 4, pp. 256-69, Apr 2007, doi: nrc2090 [pii] 10.1038/nrc2090.

[17] J. Jamnagerwalla et al., "Serum cholesterol and risk of high-grade prostate cancer: results from the REDUCE study," (in eng), Prostate Cancer Prostatic Dis, vol. 21, no. 2, pp. 252-259, Jun 2018, doi: 10.1038/s41391-017-0030-9.

[18] A. P. Carson, G. Howard, G. L. Burke, S. Shea, E. B. Levitan, and P. Muntner, "Ethnic Differences in Hypertension Incidence among MiddleAged and Older U. S. Adults: The Multi-Ethnic Study of Atherosclerosis," (in eng), Hypertension, vol. 57, no. 6, pp. 1101-7, Jun 2011, doi: 10.1161/hypertensionaha.110.168005.

[19] J. Kwagyan et al., "OBESITY AND CARDIOVASCULAR DISEASES IN A HIGH-RISK POPULATION: EVIDENCE-BASED APPROACH TO CHD RISK REDUCTION," (in eng), Ethn Dis, vol. 25, no. 2, pp. 208-13, Spring 2015. 
[20] R. L. Siegel, K. D. Miller, and A. Jemal, "Cancer statistics, 2018," (in eng), CA Cancer J Clin, vol. 68, no. 1, pp. 7-30, Jan 2018, doi: 10.3322/caac.21442.

[21] K. N. Sourbeer et al., "Metabolic syndrome-like components and prostate cancer risk: results from the Reduction by Dutasteride of Prostate Cancer Events (REDUCE) study," (in eng), BJU Int, vol. 115, no. 5, pp. 736-43, May 2015, doi: 10.1111/bju.12843.

[22] P. H. Carroll and J. L. Mohler, "NCCN Guidelines Updates: Prostate Cancer and Prostate Cancer Early Detection," (in eng), J Nat/ Compr Canc Netw, vol. 16, no. 5s, pp. 620-623, May 2018, doi: 10.6004/jnccn.2018.0036.

[23] A. R. Gaines et al., "The association between race and prostate cancer risk on initial biopsy in an equal access, multiethnic cohort," Cancer Causes Control, vol. 25, no. 8, pp. 1029-35, Aug 2014, doi: 10.1007/s10552-014-0402-6.

[24] L. A. Mucci and M. J. Stampfer, "Mounting Evidence for Prediagnostic Use of Statins in Reducing Risk of Lethal Prostate Cancer," Journal of Clinical Oncology, vol. 32, no. 1, pp. 1-2, 2014, doi: 10.1200/jco.2013.53.2770.

[25] H. Yu et al., "Effect of Metformin on Cancer Risk and Treatment Outcome of Prostate Cancer: A Meta-Analysis of Epidemiological Observational Studies," (in eng), PLoS One, vol. 9, no. 12, 2014, doi: 10.1371/journal.pone.0116327.

[26] K. S. Sfanos and A. M. De Marzo, "Prostate cancer and inflammation: the evidence," (in eng), Histopathology, vol. 60, no. 1, pp. 199-215, Jan 2012, doi: 10.1111/j.1365-2559.2011.04033.x.

[27] B. Arcidiacono et al., "Insulin resistance and cancer risk: an overview of the pathogenetic mechanisms," (in eng), Exp Diabetes Res, vol. 2012, p. 789174, 2012, doi: 10.1155/2012/789174.

[28] R. S. Mani et al., "Inflammation induced oxidative stress mediates gene fusion formation in prostate cancer," (in eng), Cell Rep, vol. 17, no. 10, pp. 2620-31, Dec 06 2016, doi: 10.1016/j.celrep.2016.11.019.

[29] F. Demichelis et al., "TMPRSS2:ERG gene fusion associated with lethal prostate cancer in a watchful waiting cohort," (in eng), Oncogene, vol. 26, no. 31, pp. 4596-9, Jul 5 2007, doi: 1210237 [pii] 10.1038/sj.onc. 1210237.

[30] A. Pettersson et al., "Modification of the association between obesity and lethal prostate cancer by TMPRSS2:ERG," J Natl Cancer Inst, vol. 105, no. 24, pp. 1881-90, Dec 18 2013, doi: 10.1093/jnci/djt332.

[31] C. K. Zhou et al., "TMPRSS2:ERG Gene Fusions in Prostate Cancer of West African Men and a Meta-Analysis of Racial Differences," (in eng), Am J Epidemiol, vol. 186, no. 12, pp. 1352-1361, Dec 15 2017, doi: 10.1093/aje/kwx235.

[32] J. Jamnagerwalla et al., "Serum cholesterol and risk of high-grade prostate cancer: results from the REDUCE study," Prostate cancer and prostatic diseases, vol. 21, no. 2, pp. 252-59, Dec 27 2018, doi: 10.1038/s41391-017-0030-9.

[33] J. Morote et al., "Role of Serum Cholesterol and Statin Use in the Risk of Prostate Cancer Detection and Tumor Aggressiveness," (in eng), Int J Mol Sci, vol. 15, no. 8, pp. 13615-23, Aug 2014, doi: 10.3390/ijms150813615.

[34] A. A. Schulman et al., "Validation of the 2015 prostate cancer grade groups for predicting long-term oncologic outcomes in a shared equalaccess health system," (in eng), Cancer, vol. 123, no. 21, pp. 4122-4129, Nov 1 2017, doi: 10.1002/cncr.30844.A

\section{Supplementary Files}

This is a list of supplementary files associated with this preprint. Click to download.

- Suppl.Table1DemographiccharacteristicsbyMSandrace.docx

- renameddbe75.docx

- Suppl.Table3DemographiccharacteristicsbyMS.docx

- renamed4a73f.docx

- renamed9898c.docx 